\title{
Deduzindo a Equação de Schrodinger Através da Analogia Óptico-Mecânica de Hamilton
}

Deducing Schrodinger's Equation Through Hamilton's Optical-Mechanical Analogy

\author{
João Quaglio ${ }^{* 1} \odot$ \\ ${ }^{1}$ Universidade Estadual de Campinas, Faculdade de Engenharia Elétrica e Computação, Campinas, SP, Brasil.
}

Recebido em 07 de junho de 2021. Revisado em 21 de julho de 2021. Aceito em 16 de agosto de 2021.

\begin{abstract}
Neste trabalho, apresentamos a analogia óptico-mecânica de Hamilton, que foi utilizada por Schrödinger para obter a famosa equação da mecânica quântica que leva seu nome. A partir desta analogia, mostramos passo a passo como obter a equação de Schrödinger (independente e dependente do tempo). Em seguida, discutimos o significado da função de onda e as interpretações propostas durante o desenvolvimento da teoria quântica. Também apresentamos soluções não difrativas para a equação de Schrödinger baseadas em soluções já conhecidas em áreas como óptica e acústica. E por fim, damos alguns exemplos de outras analogias que existem entre a óptica e a mecânica quântica.
\end{abstract}

Palavras-chave: Schrödinger, Hamilton, Analogia.

In this work, we present Hamilton's optical-mechanical analogy, which was used by Schrödinger to obtain the famous quantum mechanics equation that bears his name. From this analogy, we show step by step how to obtain Schrödinger's equation (time independent and dependent). Then, we discuss the meaning of the wave function and the proposed interpretations during the development of quantum theory. We also present non-diffractive solutions for the Schrödinger equation based on solutions already known in areas such as optics and acoustics. And finally, we give some examples of other analogies that exist between optics and quantum mechanics.

Keywords: Schrödinger, Hamilton, Analogy.

\section{Introdução}

No início do século XX, a física sofreu mudanças profundas devido ao surgimento da mecânica quântica. Da mesma forma que a mecânica relativística pode ser vista como uma generalização da mecânica clássica para altas velocidades, geralmente acima de $10 \%$ de $c$ [1], a mecânica quântica pode ser enxergada como uma generalização da mecânica clássica para dimensões muito pequenas, geralmente menores que $10^{-9} \mathrm{~m}[2]$. O termo "quântico" vem da solução proposta por Max Planck em 1900 para resolver a divergência entre a teoria e experiência da distribuição espectral da radiação térmica emitida por um corpo aquecido. Como a divergência se dá a partir de frequências no espectro ultravioleta, o problema foi chamado de "catástrofe ultravioleta". Planck sugeriu que a troca de energia no corpo não ocorria de forma contínua como sugeria a física clássica, mas sim em múltiplos inteiros de $h \nu \|^{1}$ Em 1923, Louis de Broglie propôs que da mesma maneira que a luz, antes considerada uma onda, podia apresentar propriedades corpusculares (como evidenciado pelos efeitos fotoelétrico e Compton), os elétrons, até então considerados partículas, poderiam apresentar comportamento

\footnotetext{
* Endereço de correspondência: joaoquaglio@hotmail.com

1 Planck adimitiu mais tarde que adotou esta hipótese pela necessidade de encontrar uma explicação teórica a qualquer preço.
}

ondulatório, onde o comprimento de onda associado é dado por $\lambda=h / p$. Por fim, em 1926, baseando-se na analogia óptico-mecânica formalizada por William Rowan Hamilton, Erwin Schrödinger encontrou uma equação de ondas para as ondas de matéria de de Broglie.

\section{A Analogia Óptico-Mecânica de Hamilton}

Apesar do nome, a analogia já fora estudada antes por Ibn al-Haytham (965-1037) e René Descartes (1596-1650) 3] mas foi William Rowan Hamilton em 1831 que a formalizou matematicamente, através da comparação entre a trajetória de uma partícula em um campo de forças conservativas com potencial $V(\mathbf{r})$ e a trajetória de um raio de luz em um meio com índice de refração $n(\mathbf{r})$.

Vamos considerar a trajetória de um raio luminoso representada por:

$$
d \mathbf{r}=\hat{\mathbf{u}} d s
$$

onde $d \mathbf{r}$ é um deslocamento infinitesimal ao longo de uma trajetória curva, $d s$ seu comprimento e $\hat{\mathbf{u}}$ um vetor unitário. Agora, invocando a identidade $(n \hat{\mathbf{u}})^{2}=n^{2} \mathrm{e}$ diferenciando ambos os lados em relação à $n$, temos:

$$
\hat{\mathbf{u}} \cdot d(n \hat{\mathbf{u}})=d n
$$


Sabendo que $d n$ é a variação de $n$ na direção de $d \mathbf{r}$, vem da definição do gradiente que:

$$
d n=\nabla n \cdot d \mathbf{r}
$$

e multiplicando a equação (3) por $d s / d s$ obtemos:

$$
d n=\nabla n \cdot \frac{d \mathbf{r}}{d s} d s
$$

Da equação (1), temos que $\hat{\mathbf{u}}=d \mathbf{r} / d s$, então levando este resultado na equação (4) ficamos com:

$$
d n=\nabla n \cdot \hat{\mathbf{u}} d s
$$

e comparando a equação (2) com a equação (5), temos:

$$
\hat{\mathbf{u}} \cdot d(n \hat{\mathbf{u}})=\nabla n \cdot \hat{\mathbf{u}} d s
$$

e portanto:

$$
\nabla n=\frac{d(n \hat{\mathbf{u}})}{d s}
$$

ou:

$$
\nabla n=\frac{d}{d s}\left(n \frac{d \mathbf{r}}{d s}\right)
$$

que é conhecida como equação diferencial dos raios.

No caso de uma partícula, também podemos representar sua trajetória através da equação (1) de modo que:

$$
\mathbf{v}=\frac{d \mathbf{r}}{d t}=\frac{d \mathbf{r}}{d s} \frac{d s}{d t}=v \hat{\mathbf{u}}
$$

e da segunda lei de Newton:

$$
\begin{aligned}
\frac{d \mathbf{p}}{d t} & =\frac{d \mathbf{p}}{d s} \frac{d s}{d t}=v \frac{d \mathbf{p}}{d s} \\
& =v \frac{d(m \mathbf{v})}{d s}=v \frac{d}{d s}\left(p \frac{d \mathbf{r}}{d s}\right)
\end{aligned}
$$

$\log \mathrm{O}$

$$
\frac{1}{v} \frac{d \mathbf{p}}{d t}=\frac{d}{d s}\left(p \frac{d \mathbf{r}}{d s}\right)
$$

portanto:

$$
\frac{d(p \hat{\mathbf{u}})}{d s}=\frac{d}{d s}\left(p \frac{d \mathbf{r}}{d s}\right)
$$

o que leva (por semelhança com a equação 7) a:

$$
\nabla p=\frac{d}{d s}\left(p \frac{d \mathbf{r}}{d s}\right)
$$

que é conhecida como equação diferencial das trajetórias. Comparando as equações $\sqrt{8}$ e $\sqrt{13}$, vemos que possuem exatamente a mesma forma ${ }^{2}$ Como o índice de refração

\footnotetext{
${ }^{2}$ Vale a pena mencionar que a forma $d / d s(f d \mathbf{r} / d s)$ onde $f$ é $n$ ou $p$, pode ser escrita como $\nabla f+\mathbf{a}$ onde a é um vetor que satisfaz a relação $\mathbf{a} \cdot \hat{\mathbf{u}}=\mathbf{0}$.
}

$n$ e o momento $p$ são grandezas análogas, para escrever $n$ (adimensional) em função de $p$, basta dividir $p$ pela constante $p_{0}=\sqrt{2 m E}$ que é o momento da partícula livre ${ }^{3}$ Assim, temos da conservação da energia que:

$$
E=\frac{p(\mathbf{r})^{2}}{2 m}+V(\mathbf{r})
$$

logo:

$$
p(\mathbf{r})=\sqrt{2 m[E-V(\mathbf{r})]}
$$

portanto:

$$
n(\mathbf{r})=\frac{p(\mathbf{r})}{p_{0}}=\frac{\sqrt{2 m[E-V(\mathbf{r})]}}{\sqrt{2 m E}}=\sqrt{1-\frac{V(\mathbf{r})}{E}}
$$

Isto quer dizer que a trajetória de uma partícula com energia $E$ em um campo de forças conservativas associado a um potencial $V$ é idêntica a trajetória de um raio de luz em um meio com índice de refração dado pela equação (16). Esta é a analogia apresentada por Hamilton.

\section{A Equação de Schrödinger}

Entre o final de 1925 e o começo de 1926, Schrödinger publicou uma série de quatro artigos (cerca de um por mês) sobre sua mecânica ondulatória, onde revela que talvez nossa mecânica clássica seja completamente análoga a óptica geométrica e como tal, está errada [...] portanto é preciso estabelecer uma mecânica ondulatória, e o método mais óbvio é a partir da analogia Hamiltoniana 4, 5]. Sendo assim, partindo da equação de ondas:

$$
\nabla^{2} \psi(\mathbf{r}, t)-\frac{1}{v^{2}} \frac{\partial^{2} \psi(\mathbf{r}, t)}{\partial t^{2}}=0
$$

e fazendo:

$$
\psi(\mathbf{r}, t)=A(\mathbf{r}) B(t)
$$

ficamos com:

$$
\frac{\nabla^{2} A}{A}=\frac{1}{B v^{2}} \frac{\partial^{2} B}{\partial t^{2}}
$$

Como cada lado da equação 19 "enxerga" o outro como uma constante, chamando essa constante de $-k^{2}$ e $A(\mathbf{r})=\psi(\mathbf{r})$ e $B=\psi(t)$, temos:

$$
\nabla^{2} \psi(\mathbf{r})+k^{2} \psi(\mathbf{r})=0
$$

e:

$$
\frac{\partial^{2} \psi(t)}{\partial t^{2}}+k^{2} v^{2} \psi(t)=0
$$

onde a equação 20 é conhecida como equação de Helmholtz, com $k^{2}=n^{2} k_{0}^{2}$. Usando a relação de de

\footnotetext{
${ }^{3}$ Isto vem da relação $k=n k_{0}$ onde $k=p / \hbar$ é o número de onda. Logo $p=n p_{0} \Leftrightarrow n=p / p_{0}$.
} 
Broglie, $\lambda=h / p$, onde $\lambda=2 \pi / k$, podemos mostrar que $k_{0}^{2}=p_{0}^{2} / \hbar^{2}=2 m E / \hbar^{2}$. Levando este resultado junto com a equação (16) na equação 20 , obtemos:

$$
\nabla^{2} \psi(\mathbf{r})+\left(1-\frac{V(\mathbf{r})}{E}\right)\left(\frac{2 m E}{\hbar^{2}}\right) \psi(\mathbf{r})=0
$$

e rearranjando os termos, chegamos a:

$$
\frac{-\hbar^{2}}{2 m} \nabla \psi(\mathbf{r})+V(\mathbf{r}) \psi(\mathbf{r})=E \psi(\mathbf{r})
$$

que é a equação de Schrödinger independente do tempo.

Uma vez que $\psi(t)=\exp (-i \omega t)$ é uma solução da equação 21, podemos usar as relações $\omega=k v, k=$ $2 \pi \nu / v$ e $E=h \nu$ para mostrar que $\omega=E / \hbar$, então:

$$
\psi(t)=\exp \left(-i \frac{E t}{\hbar}\right)
$$

e da equação (18):

$$
\psi(\mathbf{r}, t)=\psi(\mathbf{r}) \exp \left(-i \frac{E t}{\hbar}\right)
$$

Agora, derivando ambos os lados da equação 25 em relação a $t$, temos:

$$
i \hbar \frac{\partial \psi(\mathbf{r}, t)}{\partial t}=E \psi(\mathbf{r}, t)
$$

de modo que ao comparar ambos os lados, podemos observar que:

$$
E=i \hbar \frac{\partial}{\partial t}
$$

Assim, multiplicando ambos os lados da equação 23 por $\psi(t)$, e reescrevendo $E$ como dado pela equação (27) obtemos:

$$
\frac{-\hbar^{2}}{2 m} \nabla \psi(\mathbf{r}, t)+V(\mathbf{r}) \psi(\mathbf{r}, t)=i \hbar \frac{\partial \psi(\mathbf{r}, t)}{\partial t}
$$

que é a equação de Schrödinger dependente do tempo.

\section{Qual o significado da função de onda de Schrödinger?}

Inicialmente, Schrödinger acreditava que a função de onda era uma expressão matemática capaz de descrever com precisão as ondas de matéria de de Broglie no espaço e no tempo, e assim como na física clássica, dadas as condições iniciais, seria possível prever sua configuração futura. Isto foi motivo de orgulho para Schrödinger, afinal, pensava ter conseguido resolver o problema trazido pela física atômica [6]. Acontece, que esta interpretação não poderia estar correta, pois Werner Heisenberg havia demonstrado recentemente que ao realizarmos uma medida, a interação com o que está sendo medido provoca alterações na mesma, tornando impossível por exemplo, medir simultaneamente e com precisão, tanto a posição quanto o momento de uma partícula. Este princípio ficou conhecido como princípio da incerteza de Heisenberg.

Foi em 1928, que Max Born propôs que a função de onda estava associada a probabilidade de encontrarmos uma partícula em uma determinada região do espaço, que é dada por:

$$
P(x)=\int_{x_{1}}^{x_{2}}|\psi(x)|^{2} d x
$$

onde $|\psi(x)|^{2}$ é chamada de densidade de probabilidade. Ou seja, $P(x)$ é a probabilidade de encontrarmos uma partícula no intervalo $\left[x_{1}, x_{2}\right]$ em um instante $t$.

Esta interpretação probabilística gerou algumas questões. Ao realizar uma medida, o sistema descrito pela função de onda já possuia a configuração observada (interpretação realista) ou foi o ato de medir que obrigou o sistema a entregar uma resposta (interpretação ortodoxa)? Existem até mesmo aqueles que afirmam que trata-se de uma questão metafísica (posição agnóstica), por isso deve ser ignorada.

Para os realistas, a teoria quântica era incompleta, pois a função de onda não era capaz de descrever com precisão o sistema em questão, sendo necessário uma outra quantidade chamada de variável oculta para tal. Em 1935, Albert Einstein, Boris Podolsky, e Nathan Rosen publicaram um artigo questionando a interpretação ortodoxa. Chamado de paradoxo EPR, defendiam que o resultado de uma medida realizada em uma parte do sistema não poderia ter um efeito imediato no resultado de uma medição realizada na outra parte, independentemente da distância entre as duas partes. Isto devia-se ao fato de acreditarem que nenhuma informação poderia ser transmitida a uma velocidade superior a da luz no vácuo. Este princícpio é conhecido como localidade. Vejamos uma descrição do paradoxo proposta por David Bohm.

Imagine um méson pi neutro que decai em um elétron e um pósitron conforme abaixo:

$$
\pi^{0}=e^{-}+e^{+}
$$

Uma vez que o méson pi estava inicialmente em repouso, o elétron e o pósitron movimentam-se em sentidos opostos e pela conservação do momento angular, tanto o elétron como o pósitron possuem spin, a questão é quem possui spin up ou down.

Caso estejam separados por uma distância muito grande, 10 anos-luz por exemplo, e meçamos o spin do elétron como down, o do pósitron será up. Para os realistas, o spin do elétron era down desde o começo e apenas não sabíamos. Já para os ortodoxos, ele não era nem up nem down, foi o ato de medir que forçou o sistema a dar uma resposta. Em outras palavras, o estado do elétron é uma superposição dos estados up e down e quando uma medida é realizada, a função de onda colapsa entregando um estado. Assim, ao obtermos o spin do elétron como down, o spin do pósitron imediatamente se tornou up. Essa ação instantânea à longas distâncias 
(também conhecida como entrelaçamento quântico) era inaceitável para os realistas.

Em 1964, John Stewart Bell provou que a teoria das variáveis ocultas era imcompatível com a teoria quântica, através da seguinte desigualdade:

$$
|C(a, b)-C(a, c)| \leq 1+C(b, c)
$$

onde $a, b$ e $c$ são três vetores unitários com orientações distintas e $C$ é chamado de correlação, que é o valor esperado do produto das medidas obtidas de dois desses vetores. Em outras palavras, ele mostrou que se a desigualdade for satisfeita, ou seja, existirem variáveis ocultas, a teoria quântica não é só incompleta, mas totalmente errada 7]. Vários experimentos foram realizados nos anos 60 e 70 para testar a desigualdade de Bell, resultando no trabalho de Aspect, Grangier, e Roger [8], onde citam que nosso experimento produz a violação mais forte da desigualdade de Bell já alcançada, e em excelente acordo com a mecânica quântica. Uma vez que é uma transposição direta do esquema ideal de Einstein, Podolsky, Rosen e Bohm, o procedimento experimental é muito simples, e não precisa de medições auxiliares como em experimentos anteriores com polarizadores de canal único. Somos, portanto, levados à rejeição de teorias realistas (ou seja, "variáveis ocultas") se aceitarmos a suposição de que não há viés nas amostras detectadas, e existe suporte experimental para esta suposição. Assim, a interpretação ortodoxa, também conhecida como interpretação de Copenhague, ganhou força e é a mais aceita até hoje.

\section{Soluções Não-Difrativas para a Equação de Schrödinger}

Como vimos na seção anterior, a probabilidade de encontrarmos uma partícula (como um elétron, por exemplo) em uma região do espaço, está relacionada à função de onda e portanto, exibe comportamento ondulatório. Isto fica evidente em experimentos de dupla fenda como o de Tonomura et al. 9] em que elétrons passavam um por vez por duas fendas até atingirem um anteparo onde um padrão de interferência ondulatório era observado ${ }^{5}$ De fato, mesmo ao se propagar livremente, a função de onda que descreve uma partícula difrata, ou seja, a probabilidade de encontrar ela no espaço diminui a medida que se propaga [10]. Soluções não difrativas para equações de ondas, como ondas planas e feixes de Bessel, são conhecidas a tempos [11-13] e também existem para a equação de Schrödinger [14, 15].

\footnotetext{
4 Existe ainda uma resistência em aceitar essa interpretação por parte de alguns físicos, que recorrem a teorias de variáveis ocultas não locais, ou seja, que não obedecem a desigualdade de Bell, mas essas teorias encontram-se na marginalidade.

${ }^{5}$ Isto é feito sem observar por qual das fendas os elétrons passam. Ao observar por qual das fendas os elétrons estão passando, a função de onda colapsa e a interferência desaparece.
}

Vamos assumir uma solução do tipo:

$$
\psi(\rho, z, \phi, t)=F(\rho, z, \phi) \exp (-i \omega t)
$$

onde $\omega=E / \hbar$ e $F$ satisfaz a equação (20), que é a equação de Helmholtz. Assumindo que $F$ tenha o formato espacial de um feixe de Bessel, ou seja, $F(\rho, z, \phi)=J_{m}\left(k_{\rho} \rho\right) \exp \left(i k_{z} z+i m \phi\right)$, temos que:

$$
\psi(\rho, z, \phi, t)=J_{m}\left(k_{\rho} \rho\right) \exp \left(i k_{z} z+i m \phi-i \frac{E t}{\hbar}\right)
$$

é uma solução da equação de Schrödinger, onde $k_{\rho}$ e $k_{z}$ são os números de onda transversal e longitudinal respectivamente e $m$ é chamado de carga topológica. É importante deixar claro que os números de onda transversal e longitudinal obedecem a restrição $k^{2}=$ $k_{\rho}^{2}+k_{z}^{2}$ onde $k=p / \hbar$. Assim, temos que $k_{\rho}=p_{\rho} / \hbar$ e $k_{z}=p_{z} / \hbar$ e a equação (33) torna-se:

$$
\psi(\rho, z, \phi, t)=J_{m}\left(\rho \frac{p_{\rho}}{\hbar}\right) \exp \left(i \frac{p_{z}}{\hbar} z+i m \phi-i \frac{E t}{\hbar}\right)
$$

Na óptica, feixes de Bessel do tipo $\psi(\rho, z, \phi, t)=$ $J_{m}\left(k_{\rho} \rho\right) \exp \left(i k_{z} z+i m \phi-i \omega t\right)$ não são quadrado integráveis, isto é, carregam energia infinita, portanto não podem ser gerados experimentalmente. Porem é possível obter versões truncadas dos mesmos [16, [17] incidindo feixes gaussianos em uma abertura anular e atravessando uma lente posteriormente. Estes feixes truncados resistem à difração por uma certa distância até começarem a se degradar.

Como a equação (34) tem sua parte espacial construída a partir destes feixes de Bessel, ela também não é quadrado integrável. Assim, um arranjo similar aos feixes ópticos truncados pode ser obtido por exemplo, para elétrons, substituindo a lente convencional por uma magnética. Desta forma, a probabilidade de encontrálos no espaço não difrata por uma certa distância, e nesta região podem ser descritos pela equação (34) sem problemas.

\section{Outras Analogias}

Como vimos, a analogia óptico-mecânica de Hamilton teve um papel fundamental no desenvolvimento da teoria quântica. Desde então, várias outras analogias entre a óptica e a mecânica quântica foram desenvolvidas para tentar entender fenômenos quânticos sob uma perspectiva mais clássica da óptica.

Por exemplo, ao considerar uma solução para a equação de Helmholtz na forma $\psi(x, y, z)=$ $A(x, y, z) \exp (i k z)$ onde $A(x, y, z)$ é um envelope que varia muito lentamente na direção de propagação $z$, é possível mostrar que $A(x, y, z)$ satisfaz uma equação conhecida como equação paraxial de ondas, dada por:

$$
\nabla_{\perp}^{2} A(x, y, z)+2 i k \frac{\partial A(x, y, z)}{\partial z}=0
$$


que é análoga à equação de Schrödinger dependente do tempo. A variável $z$ ocupa o lugar de $t$, o laplaciano transversal $\nabla_{\perp}^{2}$ ocupa o lugar do laplaciano completo $\nabla^{2}$ e não existe um termo análogo ao potencial $V$ devido ao meio ser homogêneo. No caso de meios não-homogêneos, surge um termo análogo ao potencial, que é responsável pelo surgimento da refração e espalhamento [18, 19].

Existem também os chamados cristais fotônicos, que são estruturas artificiais formadas por arranjos periódicos de materiais dielétricos que afetam o movimento de fótons de maneira semelhante a que redes cristalinas afetam o movimento de elétrons. Estes cristais podem limitar a propagação de fótons com determinadas energias em regiões chamadas de bandas proibidas (ou bandgaps) fotônicas, da mesma forma que bandas proibidas limitam a movimentação de elétrons em redes cristalinas [20]. Até mesmo analogias envolvendo a relatividade foram propostas, como em [21], onde os autores mostram que para meios fracamente não-homogêneos, isto é, quando a permissividade elétrica do meio varia suavemente com a posição, as equações de Maxwell assumem uma forma paraxial semelhante à equação de Dirac.

Muitas outras analogias podem ser encontradas na literatura, e as abordadas aqui, evidenciam muito bem a importância do tema.

\section{Conclusões}

A mecânica quântica é sem dúvidas uma ferramenta poderosa para descrever sistemas físicos em uma escala inacessível para os modelos clássicos. No mundo do muito grande, a mecânica clássica é suficiente devido ao fato das massas dos objetos de estudo envolvidos serem grandes o bastante para tornar os comprimentos de onda de de Broglie muito pequenos, tornando efeitos quânticos imperceptíveis nessa escala. Quando vamos para o mundo do muito pequeno, onde as massas são muito pequenas, os comprimentos de onda de de Broglie já não podem ser ignorados, o que atribui características ondulatórias à objetos que antes eram considerados partículas. A revelação da natureza dual da matéria não só abalou a comunidade científica, como modificou profundamente o nosso entendimento da realidade.

\section{Agradecimentos}

Ao Prof. Michel Zamboni-Rached pela prontidão e pelas valiosas discussões e à Coordenação de Aperfeiçoamento de Pessoal de Ensino Superior (CAPES) pelo financiamento durante a produção deste trabalho.

\section{Referências}

[1] D. Halliday, R. Resnick e J. Walker, Fundamentos de Física: Óptica e Física Moderna (LTC, Rio de Janeiro, 2009), v. 4.
[2] H. Lüth, Quantum Physics in the Nanoworld: Schrödinger's Cat and the Dwarfs (Springer, Cham, 2009).

[3] S.A. Khan, Optik 130, 714 (2017).

[4] H.M. Nussensveig, Curso de Física Básica: Ótica, Relatividade e Física Quântica (Blucher, São Paulo, 2014), v. 4.

[5] B.R. Masters, Optics \& Photonics News 25, 32 (2014).

[6] M. Gleiser, A Dança do Universo: Dos Mitos de Criação ao Big Bang (Companhia da Letras, São Paulo, 1997).

[7] D.J. Griffiths, Introduction to Quantum Mechanics (Prentice Hall, New Jersey, 1995).

[8] A. Aspect, P. Grangier e G. Roger, Phys. Rev. Lett. 49, 91 (1982).

[9] A. Tonomura, J. Endo, T. Matsuda, T. Kawasaki e H. Ezawa, Am. J. Phys. 57, 117 (1989).

[10] T. Norsen, Foundations of Quantum Mechanics: An Exploration of the Physical Meaning of Quantum Theory (Springer, Cham, 2017).

[11] H. Bateman, Electrical and Optical Wave Motion (Cambridge University Press, Cambridge, 1915).

[12] R. Courant e D. Hilbert, Methods of Mathematical Physics (Wiley, New York, 1966).

[13] J.A. Stratton, Electromagnetic Theory (McGraw-Hill, New York, 1941).

[14] M. Zamboni-Rached e E. Recami, J. Math. Phys. 53, 052102 (2012).

[15] H.E.H. Figueroa, M. Zamboni-Rached e E. Recami, Localized Waves (Wiley, New York, 2008).

[16] J. Durnin, J.J. Miceli e J.H. Eberly, Diffraction-Free Beams, Phys. Rev. Lett. 58, 1499 (1987).

[17] M. Zamboni-Rached, E. Recami e M. Balma, Appl. Opt. 51, 3370 (2012).

[18] M.F. Souza, R. Silveira, K.Z. Nóbrega e C.A. Dartora, Revista Brasileira de Ensino de Física 36, 3308 (2014)

[19] C.A. Dartora, K.Z. Nobrega e G.G. Cabrera, Phys. Lett. A 375, 2254 (2011).

[20] T. Krauss, R. Rue e S. Brand, Nature 383, 699 (1996).

[21] M. Mehrafarin e H. Balajany, Phys. Lett. A 374, 1608 (2010). 